\title{
Comparação da mobilidade, força muscular e medo de cair em idosas caidoras e não caidoras
}

\author{
Comparison of mobility, muscle strength and fear of fall in falling and non-falling elderly
}

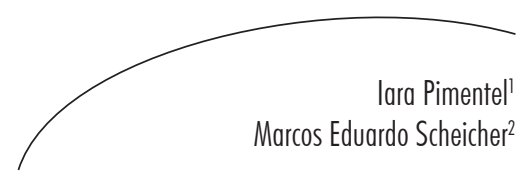

Resumo

Objetivos: Verificar o medo de cair em idosas caidoras e não caidoras ativas fisicamente e comparar mobilidade e força de preensão palmar; verificar se existe relação entre mobilidade, força muscular e medo de quedas. Métodos: Foram avaliadas 40 idosas ativas, idade $\geq 60$ anos, divididas em caidoras $(n=20)$ e não caidoras $(n=20)$. Utilizouse Mini-Exame do Estado Mental (MEEM) para rastreio cognitivo; Falls Efficacy ScaleInternational-Brasil (FES-I-BRASIL) para avaliar o medo de cair; a força muscular foi mensurada pela medida da força de preensão palmar por meio de dinamômetro hidráulico; Timed Up and Go (TUG) para avaliar mobilidade funcional. Resultados: Não foi encontrada diferença significante entre os grupos no que diz respeito à mobilidade e força muscular. A maioria das participantes $(92,5 \%)$ demonstrou preocupação com quedas. Das idosas que levaram mais que 12 segundos para desempenhar o TUG, $53,84 \%$ eram do grupo caidor. Não foi encontrada correlação entre mobilidade e força muscular. Conclusão: Não foi observada diferença significativa entre força muscular, mobilidade e medo de quedas entre os grupos. Não foi encontrada correlação significativa entre mobilidade, força muscular e medo de quedas. O estudo permitiu observar que o medo de cair está presente na maioria da população idosa, com ou sem história de quedas.

\section{Abstract}

Objectives: Check the fear of fall among falling and non falling physically active elderly and compare mobility and grip strength; verify the association between mobility, muscle strength and fear of falling. Methods: We evaluated 40 active elderly, female, age $\geq 60$ years, divided into fallers $(n=20)$ and non-fallers $(n=20)$. We used the Mini Mental State Examination (MEEM) for cognitive monitoring; Falls Efficacy Scale-

\footnotetext{
Curso de Fisioterapia, Faculdade de Filosofia e Ciências. Universidade Estadual Paulista. Marília, SP, Brasil.

2 Departamento de Fisioterapia e Terapia Ocupacional, Faculdade de Filosofia e Ciências. Universidade Estadual Paulista. Marília, SP, Brasil.

Apoio financeiro: FAPESP, Iniciação Científica, processo nº 2010/19061-1.

Correspondência / Correspondence

Marcos Eduardo Scheicher

E-mail: mscheicher@marilia.unesp.br
}

Palavras-chave:

Envelhecimento. Equilíbrio

Postural. Força Muscular. 
International-Brazil (FES-I-BRAZIL) to assess fear of falling, muscle strength was measured by measuring the strength of hand grip through the hydraulic dynamometer, Timed Up and Go (TUG) to assess functional mobility. Results: There was no significant difference between groups with regard to mobility and muscle strength. Most participants $(92.5 \%)$ expressed concern over the falls. Of the elderly who took more than 12 seconds to perform the TUG, 53.84\% were faller group. No correlation was found between mobility and muscle strength. Conclusion: No significant difference was observed between muscle strength, mobility and fear of falls among the groups. There was no significant association between mobility, muscle strength and fear of falling. This study has observed that the fear of falling is present in most of the elderly population, with or without falls history.
Key words: Aging. Postural Balance. Muscle Strength.

\section{INTRODUÇÃO}

Projeções indicam que em 2020 o Brasil ultrapassará 30 milhões de pessoas com mais de 65 anos de idade. ${ }^{1}$ Estima-se que, de 1996 a 2025, o percentual de idosos aumentará cerca de $200 \%$ nos países em desenvolvimento. ${ }^{2}$

O processo natural de envelhecimento acarreta mudanças nos vários sistemas do corpo humano, inclusive os sistemas músculoesquelético, vestibular, visual e proprioceptivo, contribuindo para prejuízos no equilíbrio postural. A instabilidade postural, somada a fatores tanto fisiológicos como patológicos, pode predispor a pessoa idosa a quedas, evento que se encontra no perfil dessa crescente população e constitui a sexta causa de morte em pessoas com mais de 65 anos de idade. Dentre os fatores que afetam a ocorrência das quedas está o gênero, sendo que mulheres caem mais do que homens. ${ }^{3,4}$

Estima-se que aproximadamente $30 \%$ dos idosos caem pelo menos uma vez ao ano. ${ }^{4}$ As consequências das quedas para os idosos, familiares e para o Sistema Único de Saúde (SUS) são preocupantes. Em 2009, foram gastos 81 milhões de reais com fraturas em idosos e no mesmo ano, ocorreram 1.478 óbitos decorrentes de fraturas de fêmur. ${ }^{5}$

Uma das consequências das quedas é o medo de cair, resultando em modificações físicas, psicológicas e sociais, que podem levar o idoso a restringir suas atividades motoras, apresentar um declínio no nível de independência e se tornar fragilizado e inseguro. ${ }^{6}$
A mobilidade é a habilidade para se mover de forma independente de um ponto para outro, devendo o indivíduo ter condições para se adaptar e modificar o andar em função de distúrbios, assim como os desafios esperados e inesperados da locomoção. ${ }^{7}$ A mobilidade ocorre graças à estabilização postural, que depende da integração entre os sistemas visual, vestibular e somato-sensorial. ${ }^{8}$ As características comumente encontradas em idosos com distúrbios da marcha e da mobilidade são: diminuição da velocidade e do comprimento do passo, perda do balanço normal dos braços e diminuição das rotações pélvica e escapular. ${ }^{9}$

O envelhecimento está associado à diminuição da massa muscular esquelética, também chamada de sarcopenia, a qual pode reduzir a força muscular e a habilidade para realizar atividades de vida diária, bem como a mobilidade. ${ }^{2} \mathrm{O}$ decréscimo na força muscular em função da idade resulta, sobretudo, da redução substancial de massa muscular que acompanha o envelhecimento, ou da diminuição da atividade física, que acaba por gerar substituição de massa muscular por gordura subcutânea..$^{10-14}$

A força muscular é um dos componentes fundamentais para avaliação da forma física. A preensão palmar pode ser considerada como um dos parâmetros do estado de força geral dos indivíduos. ${ }^{15}$ Tem sido descrita uma associação entre força de preensão palmar e desenvolvimento de incapacidades e mortalidade. ${ }^{16,17}$ A hipótese, então, é que idosas com história de quedas apresentem valores de força de preensão palmar, mobilidade e medo de cair menores do que idosas 
sem história de quedas e que há uma relação entre essas variáveis.

Os objetivos do presente estudo foram verificar o medo de cair em idosas caidoras e não caidoras fisicamente ativas e comparar mobilidade e força de preensão palmar; verificar se existe relação entre mobilidade, força muscular e medo de quedas.

\section{METODOLOGIA}

Estudo transversal, realizado entre fevereiro e outubro de 2011, onde foram avaliadas 61 idosas, das quais 21 foram excluídas devido a problemas visuais não corrigidos, uso contínuo de antidepressivos, sedativos e hipnóticos, não atingir a pontuação mínima no Mini-Exame do Estado Mental (MEEM), ser classificada como sedentária, ou devido à artrose em locais que afetassem a marcha e o equilíbrio postural. Foram incluídas apenas mulheres, pois a prevalência das quedas é maior entre elas. ${ }^{3,4}$

A amostra foi constituída então por 40 idosas distribuídas igualmente em dois grupos: caidoras e não caidoras. Todas as participantes foram classificadas como ativas fisicamente de acordo com a Sociedade Brasileira de Medicina do Esporte. ${ }^{18}$ Foi realizado o rastreio cognitivo por meio do Mini- Exame do Estado Mental, como proposto por Lourenço \& Veras. ${ }^{19}$ A idosa que relatou ter sofrido pelo menos uma queda nos 12 meses anteriores à data da avaliação inicial foi considerada caidora. ${ }^{20}$

A mobilidade funcional foi avaliada por meio do teste Timed up and go (TUG), mensurando em segundos o tempo gasto pela voluntária para levantar-se de uma cadeira com braços, andar três metros, dar a volta, retornar e sentar-se. O teste foi realizado uma vez para familiarização e uma segunda vez para tomada do tempo. ${ }^{21} \mathrm{O}$ TUG é um dos testes mais usados para avaliação da mobilidade, equilíbrio e risco de quedas em idosos. $^{22}$ As idosas que desempenharam o TUG em 12 segundos ou menos foram consideradas com mobilidade dentro da normalidade; acima de 12 segundos, a mobilidade foi considerada ruim. ${ }^{23}$
A força muscular foi mensurada pela medida da força de preensão palmar por meio de dinamômetro hidráulico, da marca North Coast Medical $^{\circledR}$, ajustado na segunda posição, a qual se considera mais adequada para esta população, considerando o tamanho da mão. As participantes foram posicionadas sentadas, com o braço aduzido paralelo ao tronco, cotovelo fletido a $90^{\circ}$ e antebraço e punho em posição neutra. Foram realizadas três medições, alternadas entre os lados dominante e não dominante, e anotado o maior valor. ${ }^{24}$ Estudos indicam que a força de preensão palmar parece manter uma relação com a força global em idosos. ${ }^{25}$

O medo de cair foi avaliado por meio da Falls Efficacy Scale-International-Brasil (FES-IBRASIL), que estima o medo de cair em 16 atividades diárias distintas, cujos valores variam de 16 pontos, para os indivíduos sem qualquer preocupação, a 64 pontos para os indivíduos com muita preocupação.

O Comitê de Ética em Pesquisa da Faculdade de Filosofia e Ciências da UNESP de Marília-SP aprovou o projeto sob $n^{\circ} 2.084 / 2010$. Todas as participantes do estudo assinaram o Termo de Consentimento Livre e Esclarecido.

Os dados foram apresentados como média \pm desvio-padrão. Após a verificação da normalidade dos dados pelo teste de Kolmogorov-Smirnov, as comparações foram feitas pelo teste de MannWhitney e as correlações pelo coeficiente de correlação de Spearman. Os seguintes valores de $\mathrm{r}$ foram adotados para identificar a força da correlação: $\mathrm{r} \leq \pm 0,5$ : correlação fraca; $\pm 0,5<$ $\mathrm{r} \leq \pm 0,7$ : correlação moderada; $0,7<\mathrm{r} \leq \pm 1$ : correlação forte. Foi adotado um valor de $\alpha \leq$ 0,05 como significante.

Neste estudo, para o cálculo do tamanho amostral mínimo, utilizou-se o método de interações com estabilização em nMínimo, ${ }^{26}$ recorrendo-se a dados da literatura das variáveis FES-I e TUG. ${ }^{27}$ Para a variável FES-I, considerouse o escore médio 24,01 ( $\mathrm{d} p=7,6)$, esperando-se obter uma diferença dessa média de no máximo 33\% (proporção do valor do escore mínimo da escala FES-I em relação ao dado da literatura de 
referência); para a variável TUG, considerando-se um valor médio de $17,73 \mathrm{~s}(\mathrm{dp}=7,58 \mathrm{~s})$, esperandose obter uma diferença dessa média de no máximo de 7,92 s (diferença entre o valor médio e o valor mínimo na amostra da literatura de referência) com uma confiança de $95 \%$ e poder do teste de $80 \%$. Obteve-se para cada grupo um tamanho amostral mínimo, estabilizado para ambas as variáveis, de 16 indivíduos.

\section{RESULTADOS}

A tabela 1 mostra as características das participantes do estudo quanto à idade, ao MEEM, à força de preensão palmar, ao TUG e ao FES-I. Não houve diferença significativa entre os grupos no que diz respeito às variáveis estudadas.

Tabela 1 - Características dos participantes do estudo. Marília, São Paulo, 2012.

\begin{tabular}{lccc}
\hline & Grupo Caidor & Grupo Não Caidor & $\alpha$ \\
\hline $\mathrm{N}$ & 20 & 20 & \\
Média de idade (anos $\pm \mathrm{DP})$ & $67.8 \pm 6.14$ & $68.75 \pm 5.04$ & 0,59 \\
Média de número de medicamentos $\pm \mathrm{DP}$ & $2.55 \pm 2.16$ & $2.5 \pm 2.03$ & 0,94 \\
MEEM \pm DP & $27.45 \pm 2.89$ & $25.9 \pm 2.97$ & 0,057 \\
Força de preensão palmar $(\mathrm{Kgf}) \pm \mathrm{DP}$ & $22.63 \pm 3.70$ & $22.73 \pm 4.87$ & 0,93 \\
TUG $(\mathrm{s}) \pm \mathrm{DP}$ & $11.60 \pm 1.76$ & $11.49 \pm 1.61$ & 0,84 \\
FES-I & $21.4 \pm 5.74$ & $26.65 \pm 8.07$ & 0,06 \\
\hline
\end{tabular}

DP: desvio padrão; MEEM: Mini Exame do Estado Mental; Kgf: kilograma-força; TUG: timed up and go; FES-I: Falls Efficacy Scale - International.

Do total de participantes, $13 \quad(32,5 \%)$ desempenharam o TUG em um tempo superior a 12 segundos, indicando menor mobilidade. Destes, sete $(53,84 \%)$ eram do grupo caidor.

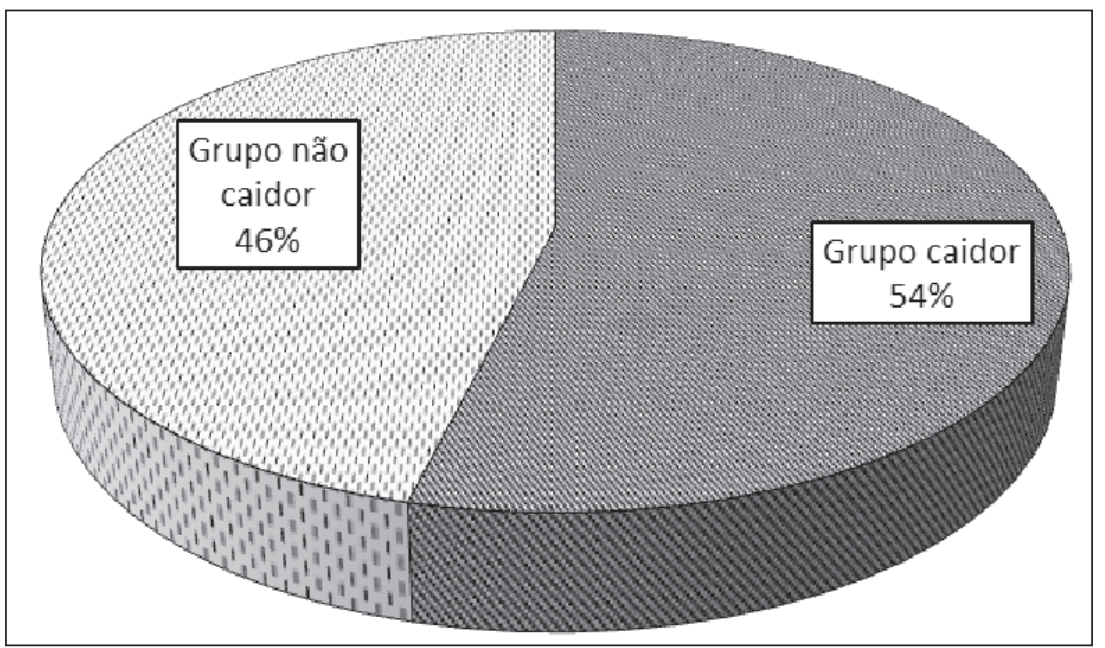

Figura 1 - Distribuição percentual de idosas que realizaram o teste TUG em mais de 12 segundos; Grupo Caidor (n = 7); Grupo Não Caidor (n=6). Marília, SP, 2011. 
A maioria das participantes (92,5\%) demonstrou preocupação com quedas em pelo menos uma das 16 tarefas propostas pela FESI-Brasil. Dentre as idosas com medo de cair, $18(48,64 \%)$ relataram histórico de queda nos últimos 12 meses. O medo de cair, portanto, foi encontrado tanto nas idosas caidoras como nas não caidoras.

No grupo não caidor houve correlação significativa entre número de medicamentos e TUG ( $r=0,32 ; \alpha=0,03)$; idade e TUG ( $r=0,55$; $\alpha=0,01)$; MEEM e TUG $(\mathrm{r}=-0,53 ; \alpha=0,01)$; idade e força de preensão palmar $(\mathrm{r}=-0,39 ; \alpha=0,01)$; idade e FES-I-Brasil ( $\mathrm{r}=0,32 ; \alpha=0,03)$; força de preensão palmar e FES-I-Brasil $(r=-0,40$; $\alpha=0,01)$; MEEM e força de preensão palmar $(\mathrm{r}=0,58 ; \alpha=0,007)$.

No grupo caidor, observou-se correlação significante entre idade e MEEM ( $\mathrm{r}=-0,49$; $\alpha=0,02 ;)$; e MEEM e TUG ( $r=-0,53 ; \alpha=0,01)$.

\section{DISCUSSÃO}

Os objetivos do presente estudo foram avaliar e comparar mobilidade, força de preensão palmar e medo de cair em idosas caidoras e não caidora, ativas fisicamente, e verificar se existe relação entre mobilidade, força muscular e medo de quedas.

A maioria das participantes do estudo relatou medo de cair (92,5\%) em pelo menos uma das atividades propostas pela FES-I Brasil, das quais $18(48,64 \%)$ relataram ocorrência de quedas. Esperava-se que o grupo de idosas com história de quedas apresentasse maior medo de cair. Observou-se, porém, que o grupo caidor referiu, em média, menos medo de cair que o grupo não caidor. Uma explicação para esse fato é que o medo de quedas está associado com fatores ambientais e psicológicos e não somente com fatores físicos (quedas x não quedas). Freitas \& Scheicher ${ }^{28}$ encontraram que $91,5 \%$ de idosos apresentaram medo de quedas nas atividades avaliadas pelo FES-I. Lopes et al. ${ }^{27}$ encontraram uma prevalência de medo de cair de 90,48\%, dentre os quais, 54,4\% relataram histórico de quedas. De acordo com Halvarsson et al., ${ }^{29}$ o medo de cair é comum após a queda, mas uma queda anterior não é necessária para desencadear o medo. Zijlstra et al. ${ }^{30}$ afirmam que o medo de cair é relatado tanto por idosos que sofreram queda, como por aqueles sem experiência anterior de quedas.

Os resultados obtidos demonstram que, no grupo caidor, o tempo médio gasto para executar o TUG foi de 11,6 segundos, e no grupo não caidor, de 11,49 segundos. Lopes et al. ${ }^{27}$ encontraram que $78,9 \%$ dos idosos que participaram de seu estudo executaram o teste entre 10 e 20 segundos. E dentre as idosas que desempenharam o TUG em um tempo superior a 12 segundos, sete $(53,84 \%)$ pertenciam ao grupo caidor, sugerindo que estas idosas tiveram pior mobilidade quando comparadas à idosas não caidoras.

Em relação à força muscular, houve correlação significante entre idade e força de preensão palmar para o grupo não caidor. Quando medida depois da quinta década de vida, a taxa de progressão da redução da força se dá em torno de 8 a $15 \%$ por década, e tanto homens quanto mulheres exibem o mesmo padrão de diminuição da força durante o envelhecimento. ${ }^{11,13}$ Não foi encontrada diferença significativa entre a média de força de preensão palmar para os grupos. Gomes et al., ${ }^{31}$ porém, encontraram que os idosos que apresentaram menor força de preensão manual foram os que relataram ocorrência de duas ou mais quedas. É preciso questionar se, realmente, a força de preensão palmar representa a força muscular de todo o organismo.

Uma das limitações do estudo foi a quantidade de participantes, devido à dificuldade de se encontrar idosos com história de quedas não relacionadas com problemas de saúde. Outra questão é que apenas mulheres foram incluídas, não sendo possível extrapolar os resultados para os homens. Mais estudos, portanto, devem ser feitos com a inclusão de mais idosos e do sexo masculino. 


\section{CONCLUSÃO}

Os resultados indicaram não haver diferença significativa entre mobilidade, força de preensão palmar e medo de cair entre idosas caidoras e não caidoras, ativas fisicamente. Além disso, observouse que o medo de cair está presente no idoso, independentemente do histórico de quedas e da prática de atividades físicas regulares, tornando-se importante a avaliação desta população.

\section{REFERÊNCIAS}

1. Veras R. Envelhecimento populacional contemporâneo: demandas, desafios e inovações. Rev Saúde Pública 2009;43(3):548-54.

2. Silva TAA, Frisoli Junior A, Pinheiro MM, Szejnfeld VL. Sarcopenia associada ao envelhecimento: aspectos etiológicos e opções terapêuticas. Rev Bras Reumatol 2006;46(6):391-7.

3. Pereira SRM, Buksman S, Perracini M, Py L, Barreto KML, Leite VMM. Quedas em idosos. Projeto Diretrizes. Associação Médica Brasileira e Conselho Federal de Medicina. Revista AMRIGS 2004;48(1):43-65.

4. Siqueira FV, Facchini LA, Piccini RX, Tomasi E, Thumé E, Silveira DS, et al. Prevalência de quedas em idosos e fatores associados. Rev Saúde Pública 2007;41(5):749-56.

5. BRASIL. Ministério da Saúde [homepage na internet]. Brasília; 2009 [acesso em 14 jul 2010]. SUS gasta quase $\mathrm{R} \$ 81$ milhões com fraturas em idosos em 2009; [1 tela]. Disponível em: <http:// portal.saude.gov.br/portal/saude/visualizar_texto. cfm?idtxt $=33674 \&$ janela $=1>$

6. Teixeira DC, Prado Junior SRR, Lima DF, Gomes SC, Brunetto AF. Efeitos de um programa de exercício físico para idosas sobre variáveis neuromotoras, antropométrica e medo de cair. Rev Bras Educ Fís Esp 2007;21(2):107-20.

7. Matsudo SM, Matsudo VKR, Barros Neto TL. Atividade física e envelhecimento: aspectos epidemiológicos. Rev Bras Med Esporte 2001;7(1):2-13.

8. Alfieri FM, Werner A, Roschel AB, Melo FC, Santos KLS. Mobilidade funcional de idosos ativos e sedentários versus adultos sedentários. Brazilian Journal of Biomotricity [periódico na Internet]. 2009 [Acesso em 2010 jul 23];3(1):89-94. Disponível em: http://www. brjb.com.br/files/brjb_66_3200903_id2.pdf.

9. Maciel ACC, Guerra RO. Fatores associados à alteração da mobilidade em idosos residentes na comunidade. Rev Bras Fisioter 2005;9(1):17-23.

10. Wilmore JH, Costill DL. Physiology of sport and exercise. Champaign, Illinois: Human Kinetics, 1999.

11. Deschenes MR. Effects of aging on muscle fibre type and size. Sports Med 2004;34(12):809-24.

12. Hunter GR, McCarthy JP, Bamman MM. Effects of resistance training on older adults. Sports Med 2004;34(5):329-48.

13. Kauffman TL. Manual de reabilitação geriátrica. Rio de Janeiro: Guanabara Koogan, 2001.

14. Rossi E, Sader CS. Envelhecimento do Sistema Osteomuscular. In: Freitas EV, Py L, Neri AL, Cançado FAX, Gorzoni ML, Rocha SM, editores. Tratado de Geriatria e Gerontologia. Rio de Janeiro: Guanabara Koogan; 2002. p. 508-14.

15. Moreira D, Alvarez RRA, Gogoy JR, Cambraia AN. Abordagem sobre preensão palmar utilizando o dinamômetro Jamar: uma revisão de literatura. RBCM 2003;11(2):95-9.

16. Ling CH, Taekema D, de Craen AJ, Gussekloo J, Westendorp RG, Maier AB. Handgrip strength and mortality in the oldest old population: the Leiden $85-$ plus study. CMAJ 2010;182(5):429-35.

17. den Ouden ME, Schuurmans MJ, Arts IE, van der Schouw YT. Physical performance characteristics related to disability in older persons: a systematic review. Maturitas 2011;69(3):208-19.

18. Carvalho T, Nóbrega ACL, Lazzoli JK, Magni JRT, Rezende L, Drummond FA, et al. Posicionamento oficial da Sociedade Brasileira de Medicina do Esporte sobre atividade física e saúde. Rev Bras Med Esporte 1996;2(4):79-81.

19. Lourenço RA, Veras RP. Mini-Exame do Estado Mental: características psicométricas em idosos ambulatoriais. Rev Saúde Pública 2006;40(4):712-19.

20. Teixeira LF. Nível de atividade física e quedas em idosos da comunidade: um estudo exploratório [dissertação de Mestrado]. São Paulo: Universidade Cidade de São Paulo; 2011.

21. Podsiadlo D, Richardson S. The Timed "Up \& GO": a test of basic functional mobility for frail elderly persons. J Am Geriatr Soc 1991;39(2):142-8. 
22. Shimada H, Suzukaw M, Tiedemann A, Kobayashi K, Yoshida H, Suzuki T. Which neuromuscular or cognitive test is the optimal screening tool to predict falls in frail community-dwelling older people? Gerontology 2009;55(5):532-8.

23. Bischoff HA, Stahelin HB, Monsch AU, Iversen MD, Weyh A, von Dechend M, et al. Identifying a cut-off point for normal mobility: a comparison of the timed 'up and go' test in community-dwellingand institutionalised elderly women. Age Ageing 2003;32(3):315-20.

24. Caporrino FA, Faloppa F, Santos JBG, Réssio C, Soares FHC, Nakachima LR, et al. Estudo populacional da força de preensão palmar com dinamômetro Jamar. Rev Bras Ortop 1998;33(2):150-4.

25. Bohannon RW. Hand-grip dynamometry predicts future outcomes in aging adults. J Geriatr Phys Ther 2008;31(1):3-10.

26. Callegari-Jacques SM. Bioestatística: princípios e aplicações. Porto Alegre: ArtMed; 2003. 256 p.

27. Lopes KT, Costa DF, Santos LF, Castro DP, Bastone AC. Prevalência do medo de cair em uma população de idosos da comunidade e sua correlação com mobilidade, equilíbrio dinâmico, risco e história de quedas. Rev Bras Fisioter 2009;13(3):223-9.

28. Freitas MAV, Scheicher ME. Preocupação de idosos em relação a quedas. Rev Bras Geriatr Gerontol 2008;11:57-64.

29. Halvarsson A, Oddsson L, Olsson E, Farén E, Pettersson A, Ståhle A. Effects of new, individually adjusted, progressive balance group training for elderly people with fear of falling and tend to fall: a randomized controlled trial. Clin Rehabil 2011;25(11):1021-31.

30. Zijlstra GA, Tennstedt SL, van Haastregt JC, van Eijk JT, Kempen GI. Reducing fear of falling and avoidance of activity in elderly persons: The development of a Dutch version of an American intervention. Patient Educ Couns 2006;62(2):220-7.

31. Gomes GAO, Cintra FA, Diogo MJD, Neri AL, Guariento ME, Sousa MLR. Comparação entre idosos que sofreram quedas segundo desempenho físico e número de ocorrências. Rev Bras Fisioter 2009;13(5):430-7. 